\title{
Existence of Positive Solutions for Neumann Boundary Value Problem with a Variable Coefficient
}

\author{
Dongming Yan, ${ }^{1}$ Qiang Zhang, ${ }^{2}$ and Zhigang $\operatorname{Pan}^{1}$ \\ ${ }^{1}$ Department of Mathematics, Sichuan University, Chengdu, China \\ ${ }^{2}$ School of Computer Science, Civil Aviation Flight University of China, Guanghan, China \\ Correspondence should be addressed to Dongming Yan, yandong_ming@126.com
}

Received 25 May 2011; Accepted 27 July 2011

Academic Editor: Bashir Ahmad

Copyright (C) 2011 Dongming Yan et al. This is an open access article distributed under the Creative Commons Attribution License, which permits unrestricted use, distribution, and reproduction in any medium, provided the original work is properly cited.

We consider the existence of positive solutions for the Neumann boundary value problem $x^{\prime \prime}(t)+$ $m^{2}(t) x(t)=f(t, x(t))+e(t), t \in(0,1), x^{\prime}(0)=0, x^{\prime}(1)=0$, where $m \in C([0,1],(0,+\infty)), e \in$ $C[0,1]$, and $f:[0,1] \times(0,+\infty) \rightarrow[0,+\infty)$ is continuous. The theorem obtained is very general and complements previous known results.

\section{Introduction}

The existence of solutions of Neumann boundary value problem of second-order ordinary differential equations has been studied by many authors; see Sun et al. [1], Cabada and Pouso [2], Cabada et al. [3], Canada et al. [4], Chu et al. [5], Jiang, and Liu [6], Yazidi [7], Sun and $\mathrm{Li}[8]$ and the references therein.

Recently, Chu et al. [5] have studied the existence of positive solution to the Neumann boundary value problem

$$
\begin{aligned}
x^{\prime \prime}(t)+m^{2} x(t) & =f(t, x(t))+e(t), \quad t \in(0,1), \\
x^{\prime}(0) & =0, \quad x^{\prime}(1)=0,
\end{aligned}
$$

where $m \in(0, \pi / 2)$ is a constant, $e \in C[0,1]$ and nonlinearity $f(t, x)$ may be singular at $x=0$. Their approach was based upon the nonlinear alternative principle of Leray-Schauder 
and Green's function, $G_{1}(t, s)$, of the associated linear problem

$$
\begin{gathered}
x^{\prime \prime}(t)+m^{2} x(t)=0, \quad t \in(0,1), \\
x^{\prime}(0)=0, \quad x^{\prime}(1)=0 .
\end{gathered}
$$

Notice that Green's function $G_{1}(t, s)$ can be explicitly expressed by

$$
G_{1}(t, s)= \begin{cases}\frac{\cos m(1-s) \cos m t}{m \sin m}, & 0 \leq t \leq s \leq 1 \\ \frac{\cos m(1-t) \cos m s}{m \sin m}, & 0 \leq s \leq t \leq 1\end{cases}
$$

In this paper, we will consider the more general problem

$$
\begin{aligned}
x^{\prime \prime}(t)+m^{2}(t) x(t) & =f(t, x(t))+e(t), \quad t \in(0,1), \\
x^{\prime}(0) & =0, \quad x^{\prime}(1)=0,
\end{aligned}
$$

where $m \in C([0,1],(0,+\infty)), e \in C[0,1]$, and $f:[0,1] \times(0,+\infty) \rightarrow[0,+\infty)$ is continuous.

Of course, the natural question is what would happen when the constant $m$ in (1.1) is replaced with a function $m(t)$ ? Obviously, Green's function of the associated linear problem

$$
\begin{gathered}
x^{\prime \prime}(t)+m^{2}(t) x(t)=0, \quad t \in(0,1), \\
x^{\prime}(0)=0, \quad x^{\prime}(1)=0
\end{gathered}
$$

cannot be explicitly expressed by elementary functions! The primary contribution of this paper is to construct Green's function associated with the Neumann boundary value problem with a variable coefficient (1.5) and study the properties of the Green's function. We apply the Krasnoselskii and Guo fixed point theorem as an application. This application was first made by Erbe and Wang [9] to ordinary differential equations. Since that time, there has been a tremendous amount of work to study the existence of positive solutions to BVPs for ordinary differential equations. Once we obtain Theorem 2.2, many of those applications would work here as well.

The rest of the paper is organized as follows: Section 2 is devoted to constructing Green's function and proves some preliminary results. In Section 3, we state and prove our main results. In Section 4, an example illustrates the applicability of the main existence result.

\section{Preliminaries and Lemmas}

Let us fix some notation to be used. Given $\varphi \in L^{1}[0,1]$, we write $\varphi>0$ if $\varphi \geq 0$ for a.e. $t \in[0,1]$, and it is positive in a set of positive measure. Let us denote by $p^{*}$ and $p_{*}$ the essential supremum and infimum of a given function $p \in L^{1}[0,1]$ if they exist. To study the boundary value problem (1.4), we need restriction on $m(t)$

$$
\left(H_{0}\right) m \in C[0,1] \text {, and } 0<\underline{m}:=\inf _{t \in[0,1]} m(t), \bar{m}:=\sup _{t \in[0,1]} m(t)<\pi / 2 .
$$


To rewrite (1.4) to an equivalent integral equation, we need to construct Green's function of the corresponding linear problem. To do this, we need the following.

Lemma 2.1. Let $\left(H_{0}\right)$ hold. Suppose $\varphi$ and $\psi$ be the solution of the linear problems

$$
\begin{gathered}
\varphi^{\prime \prime}(t)+m^{2}(t) \varphi(t)=0, \quad t \in[0,1], \\
\varphi^{\prime}(0)=0, \quad \varphi(0)=1, \\
\psi^{\prime \prime}(t)+m^{2}(t) \psi(t)=0, \quad t \in[0,1], \\
\psi^{\prime}(1)=0, \quad \psi(1)=1,
\end{gathered}
$$

respectively. Then

(i) $\varphi(t)>0$ on $[0,1]$, and $\varphi^{\prime}(t)<0$ on $(0,1]$;

(ii) (ii) $\psi(t)>0$ on $[0,1]$, and $\psi^{\prime}(t)>0$ on $[0,1)$.

Proof. We will give a proof for (i) only. The proof of (ii) follows in a similar manner.

It is easy to see that the problem

$$
\begin{gathered}
x^{\prime \prime}(t)+\bar{m}^{2} x(t)=0, \quad t \in[0,1], \\
x^{\prime}(0)=0, \quad x(0)=1
\end{gathered}
$$

has the unique solution $x(t)=\cos \bar{m} t$ and $t \in[0,1]$. From $\left(H_{0}\right)$, we know that

$$
\cos \bar{m} t>0, \quad t \in[0,1] .
$$

On the other hand, for all $t \in[0,1]$, we have

$$
(\cos \bar{m} t)^{\prime \prime}+m^{2}(t) \cos \bar{m} t=-\bar{m}^{2} \cos \bar{m} t+m^{2}(t) \cos \bar{m} t=\left(m^{2}(t)-\bar{m}^{2}\right) \cos \bar{m} t \leq 0
$$

By using comparison theorem (see [10]), we obtain

$$
\cos \bar{m} t \leq \varphi(t), \quad t \in[0,1] .
$$

Therefore, we have from (2.3) and (2.5) that

$$
0<\cos \bar{m} t \leq \varphi(t), \quad t \in[0,1]
$$

Thus

$$
\varphi^{\prime \prime}(t)=-m^{2}(t) \varphi(t)<0, \quad t \in[0,1]
$$

From the fact $\varphi^{\prime}(0)=0$ and $(2.7)$, we obtain $\varphi^{\prime}(t)<0$ on $(0,1]$. 
Now, let

$$
G(t, s)=\frac{1}{\psi^{\prime}(0)} \begin{cases}\psi(t) \varphi(s), & 0 \leq s \leq t \leq 1 \\ \psi(s) \varphi(t), & 0 \leq t \leq s \leq 1\end{cases}
$$

Theorem 2.2. Let $\left(H_{0}\right)$ hold. Then for any $y \in C[0,1]$, the problem

$$
\begin{gathered}
x^{\prime \prime}(t)+m^{2}(t) x(t)=y(t), \quad t \in(0,1), \\
x^{\prime}(0)=0, \quad x^{\prime}(1)=0
\end{gathered}
$$

is equivalent to the integral equation

$$
x(t)=\int_{0}^{1} G(t, s) y(s) d s .
$$

Proof. First we show that the unique solution of (2.9) can be represented by (2.10).

In fact, we know that the equation

$$
x^{\prime \prime}(t)+m^{2}(t) x(t)=0, \quad t \in[0,1]
$$

has known two linear independent solutions $\varphi$ and $\psi$ since $\left|\begin{array}{cc}\varphi(0) & \psi(0) \\ \varphi^{\prime}(0) & \psi^{\prime}(0)\end{array}\right|=\psi^{\prime}(0) \neq 0$.

Now by the method of variation of constants, we can obtain that the unique solution of the problem (2.9) can be represented by

$$
x(t)=\int_{0}^{1} G(t, s) y(s) \mathrm{d} s,
$$

where $G(t, s)$ is as $(2.8)$.

Next we check that the function defined by (2.10) is a solution of (2.9).

From (2.10), we know that

$$
\begin{gathered}
x(t)=\int_{0}^{t} \frac{\psi(t) \varphi(s)}{\psi^{\prime}(0)} y(s) \mathrm{d} s+\int_{t}^{1} \frac{\psi(s) \varphi(t)}{\psi^{\prime}(0)} y(s) \mathrm{d} s, \\
x^{\prime}(t)=\psi^{\prime}(t) \int_{0}^{t} \frac{\varphi(s)}{\psi^{\prime}(0)} y(s) \mathrm{d} s+\varphi^{\prime}(t) \int_{t}^{1} \frac{\psi(s)}{\psi^{\prime}(0)} y(s) \mathrm{d} s, \\
x^{\prime \prime}(t)=\psi^{\prime \prime}(t) \int_{0}^{t} \frac{\varphi(s)}{\psi^{\prime}(0)} y(s) \mathrm{d} s+\varphi^{\prime \prime}(t) \int_{t}^{1} \frac{\psi(s)}{\psi^{\prime}(0)} y(s) \mathrm{d} s+\frac{\psi^{\prime}(t) \varphi(t)}{\psi^{\prime}(0)} y(t)-\frac{\varphi^{\prime}(t) \psi(t)}{\psi^{\prime}(0)} y(t) .
\end{gathered}
$$


So that

$$
\begin{aligned}
x^{\prime \prime}(t)+m^{2}(t) x(t) & =\frac{1}{\psi^{\prime}(0)}\left|\begin{array}{cc}
\varphi(t) & \psi(t) \\
\varphi^{\prime}(t) & \psi^{\prime}(t)
\end{array}\right| y(t) \\
& =\frac{1}{\psi^{\prime}(0)}\left|\begin{array}{cc}
\varphi(0) & \psi(0) \\
\varphi^{\prime}(0) & \psi^{\prime}(0)
\end{array}\right| y(t)=y(t) .
\end{aligned}
$$

Finally, it is easy to see that

$$
x^{\prime}(0)=\varphi^{\prime}(0) \int_{0}^{1} \frac{\psi(s)}{\psi^{\prime}(0)} y(s) \mathrm{d} s=0, \quad x^{\prime}(1)=\psi^{\prime}(1) \int_{0}^{1} \frac{\varphi(s)}{\psi^{\prime}(0)} y(s) \mathrm{d} s=0 .
$$

From Lemma 2.1, we know that

$$
G(t, s)>0, \quad \forall t, s \in[0,1]
$$

Let $A=\min _{0 \leq t, s \leq 1} G(t, s), B=\max _{0 \leq t, s \leq 1} G(t, s), \sigma=A / B$. Then $B>A>0$ and $0<\sigma<1$.

In order to prove the main result of this paper, we need the following fixed-point theorem of cone expansion-compression type due to Krasnoselskii's (see [11]).

Theorem 2.3. Let $E$ be a Banach space, and $K \subset E$ is a cone in $E$. Assume that $\Omega_{1}$ and $\Omega_{2}$ are open subsets of $E$ with $\theta \in \Omega_{1}$ and $\bar{\Omega}_{1} \subset \Omega_{2}$. Let $T: K \cap\left(\bar{\Omega}_{2} \backslash \Omega_{1}\right) \rightarrow K$ be a completely continuous operator. In addition, suppose that either

(i) $\|T u\| \leq\|u\|, \forall u \in K \cap \partial \Omega_{1}$ and $\|T u\| \geq\|u\|, \forall u \in K \cap \partial \Omega_{2}$ or

(ii) $\|T u\| \geq\|u\|, \forall u \in K \cap \partial \Omega_{1}$ and $\|T u\| \leq\|u\|, \forall u \in K \cap \partial \Omega_{2}$ holds.

Then $T$ has a fixed point in $K \cap\left(\bar{\Omega}_{2} \backslash \Omega_{1}\right)$.

\section{Main Results}

In this section, we state and prove the main results of this paper.

Let us define the function

$$
r(t)=\int_{0}^{1} G(t, s) e(s) \mathrm{d} s
$$

which is just the unique solution of the linear problem (2.9) with $y(t)=e(t)$. For our constructions, let $E=C[0,1]$, with norm $\|x\|=\sup _{0 \leq t \leq 1}|x(t)|$. Define a cone $P$, by

$$
P=\left\{x \in E \mid x(t) \geq 0 \text { on }[0,1], \text { and } \min _{0 \leq t \leq 1} x(t) \geq \sigma\|x\|\right\}
$$


Theorem 3.1. Let $\left(H_{0}\right)$ hold. Suppose that there exist a constant $r>0$ such that

$\left(H_{1}\right)$ there exist continuous, nonnegative functions $g, h$, and $k$, such that

$$
0 \leq f(t, x) \leq k(t)[g(x)+h(x)] \quad \forall(t, x) \in[0,1] \times(0, r]
$$

$g(x)>0$ is nonincreasing, and $h(x) / g(x)$ is nondecreasing in $x \in(0, r]$;

$\left(H_{2}\right) r-r^{*} /(g(\sigma r)(1+h(r) / g(r)))>K^{*}$, here $K(t)=\int_{0}^{1} G(t, s) k(s) d s ;$

$\left(H_{3}\right)$ there exists a continuous function $\phi_{r}>0$ such that

$$
f(t, x) \geq \phi_{r}(t) \quad \forall(t, x) \in[0,1] \times(0, r] ;
$$

$\left(H_{4}\right) \phi_{r}(t)+e(t)>0 \quad$ for all $t \in[0,1]$.

Then problem (1.4) has at least one positive solution $x$ with $0<\|x\|<r$.

Remark 3.2. When $m(t) \equiv m, t \in[0,1]$, then (1.4) reduces to (1.1), $\left(H_{0}\right)$ reduce to $m \in$ $(0, \pi / 2)$. So Theorem 3.1 is more extensive than [5, Theorem 3.1].

Proof of Theorem 3.1. Let $\delta=\min _{0 \leq t \leq 1} \int_{0}^{1} G(t, s) \phi_{r}(s) \mathrm{d} s+\gamma_{*}$. Choose $n_{0} \in\{1,2, \ldots\}$ such that $1 / n_{0}<\sigma r_{1}$, where $0<r_{1}<\min \{\delta, r\}$ is a constant. Let $N_{0}=\left\{n_{0}+1, n_{0}+2, \ldots\right\}$. Fix $n \in N_{0}$. Consider the boundary value problem

$$
\begin{aligned}
x^{\prime \prime}(t)+m^{2}(t) x(t) & =f_{n}(t, x(t))+e(t), \quad t \in(0,1), \\
x^{\prime}(0) & =0, \quad x^{\prime}(1)=0,
\end{aligned}
$$

where

$$
f_{n}(t, x)= \begin{cases}f(t, x), & \text { if } x \geq \frac{1}{n} \\ f\left(t, \frac{1}{n}\right), & \text { if } 0 \leq x \leq \frac{1}{n}\end{cases}
$$

We note that $x$ is a solution of $\left(3.1_{n}\right)$ if and only if

$$
x(t)=\int_{0}^{1} G(t, s)\left[f_{n}(s, x(s))+e(s)\right] \mathrm{d} s, \quad 0 \leq t \leq 1 .
$$

Define an integral operator $T_{n}: P \rightarrow E$ by

$$
\left(T_{n} x\right)(t)=\int_{0}^{1} G(t, s)\left[f_{n}(s, x(s))+e(s)\right] \mathrm{d} s, \quad 0 \leq t \leq 1, x \in P .
$$

Then, $\left(3.1_{n}\right)$ is equivalent to the fixed point equation $x=T_{n} x$. We seek a fixed point of $T_{n}$ in the cone $P$. 
International Journal of Differential Equations

$$
\begin{gathered}
\text { Set } \Omega_{1}=\left\{x \in E \mid\|x\|<r_{1}\right\}, \Omega_{2}=\{x \in E \mid\|x\|<r\} \text {. If } x \in P \cap\left(\bar{\Omega}_{2} \backslash \Omega_{1}\right) \text {, then } \\
r \geq x(t) \geq \sigma\|x\| \geq \sigma r_{1}>\frac{1}{n_{0}}>\frac{1}{n}>0 \text { on }[0,1] .
\end{gathered}
$$

Notice from (2.16), $\left(H_{3}\right)$, and $\left(H_{4}\right)$ that, for $x \in P \cap\left(\bar{\Omega}_{2} \backslash \Omega_{1}\right),\left(T_{n} x\right)(t) \geq 0$ on [0,1]. Also, for $x \in P \cap\left(\bar{\Omega}_{2} \backslash \Omega_{1}\right)$, we have

$$
\begin{aligned}
\left(T_{n} x\right)(t) & =\int_{0}^{1} G(t, s)\left[f_{n}(s, x(s))+e(s)\right] \mathrm{d} s \\
& \leq \max _{0 \leq t, s \leq 1} G(t, s) \int_{0}^{1}\left[f_{n}(s, x(s))+e(s)\right] \mathrm{d} s, \quad t \in[0,1],
\end{aligned}
$$

so that

$$
\left\|T_{n} x\right\| \leq \max _{0 \leq t, s \leq 1} G(t, s) \int_{0}^{1}\left[f_{n}(s, x(s))+e(s)\right] \mathrm{d} s .
$$

And next, if $x \in P \cap\left(\bar{\Omega}_{2} \backslash \Omega_{1}\right)$, we have by (3.10),

$$
\begin{aligned}
\min _{0 \leq t \leq 1}\left(T_{n} x\right)(t) & =\min _{0 \leq t \leq 1} \int_{0}^{1} G(t, s)\left[f_{n}(s, x(s))+e(s)\right] \mathrm{d} s \\
& \geq \min _{0 \leq t, s \leq 1} G(t, s) \int_{0}^{1}\left[f_{n}(s, x(s))+e(s)\right] \mathrm{d} s \\
& =\sigma \max _{0 \leq t, s \leq 1} G(t, s) \int_{0}^{1}\left[f_{n}(s, x(s))+e(s)\right] \mathrm{d} s \\
& \geq \sigma\left\|T_{n} x\right\| .
\end{aligned}
$$

As a consequence, $T_{n}: P \cap\left(\bar{\Omega}_{2} \backslash \Omega_{1}\right) \rightarrow P$. In addition, standard arguments show that $T_{n}$ is completely continuous.

If $x \in P$ with $\|x\|=r$, then

$$
r \geq x(t) \geq \sigma\|x\|=\sigma r>\sigma r_{1}>\frac{1}{n_{0}}>\frac{1}{n}>0 \text { on }[0,1]
$$


and we have by $\left(H_{1}\right),\left(H_{2}\right)$, and $\left(H_{3}\right)$

$$
\begin{aligned}
\left(T_{n} x\right)(t) & =\int_{0}^{1} G(t, s)\left[f_{n}(s, x(s))+e(s)\right] \mathrm{d} s \\
& \leq \int_{0}^{1} G(t, s) k(s)[g(x(s))+h(x(s))] \mathrm{d} s+r^{*} \\
& \leq g(\sigma r)\left(1+\frac{h(r)}{g(r)}\right) K^{*}+r^{*} \\
& <r=\|x\|, \quad t \in[0,1] .
\end{aligned}
$$

Thus, $\left\|T_{n} x\right\| \leq\|x\|$. Hence,

$$
\left\|T_{n} x\right\| \leq\|x\|, \quad \text { for } x \in P \cap \partial \Omega_{2} .
$$

If $x \in P$ with $\|x\|=r_{1}$, then

$$
r>r_{1} \geq x(t) \geq \sigma\|x\|=\sigma r_{1}>\frac{1}{n_{0}}>\frac{1}{n}>0 \text { on }[0,1],
$$

and we have by $\left(H_{3}\right)$ and $\left(H_{4}\right)$

$$
\begin{aligned}
\left(T_{n} x\right)\left(\frac{1}{2}\right) & =\int_{0}^{1} G\left(\frac{1}{2}, s\right)\left[f_{n}(s, x(s))+e(s)\right] \mathrm{d} s \\
& \geq \int_{0}^{1} G\left(\frac{1}{2}, s\right)\left[\phi_{r}(s)+e(s)\right] \mathrm{d} s \\
& \geq \min _{0 \leq t \leq 1} \int_{0}^{1} G(t, s)\left[\phi_{r}(s)+e(s)\right] \mathrm{d} s \\
& \geq \min _{0 \leq t \leq 1} \int_{0}^{1} G(t, s) \phi_{r}(s) \mathrm{d} s+\gamma_{*} \\
& =\delta>r_{1}=\|x\| .
\end{aligned}
$$

Thus, $\left\|T_{n} x\right\| \leq\|x\|$. Hence,

$$
\left\|T_{n} x\right\| \leq\|x\|, \quad \text { for } x \in P \cap \partial \Omega_{1} .
$$

Applying (ii) of Theorem 2.3 to (3.14) and (3.17) yields that $T_{n}$ has a fixed point $x_{n} \in$ $P \cap\left(\bar{\Omega}_{2} \backslash \Omega_{1}\right)$, and $r_{1} \leq\left\|x_{n}\right\| \leq r$. As such, $x_{n}$ is a solution of $\left(3.1_{n}\right)$, and

$$
r \geq x_{n}(t) \geq \sigma\left\|x_{n}\right\| \geq \sigma r_{1}>\frac{1}{n_{0}}>\frac{1}{n}>0, \quad t \in[0,1] .
$$


Next we prove the fact

$$
\left\|x_{n}^{\prime}\right\| \leq H
$$

for some constant $H>0$ and for all $n>n_{0}$. To this end, integrating the first equation of $\left(3.1_{n}\right)$ from 0 to 1 , we obtain

$$
\int_{0}^{1} m^{2}(t) x_{n}(t) \mathrm{d} t=\int_{0}^{1}\left[f_{n}\left(t, x_{n}(t)\right)+e(t)\right] \mathrm{d} t
$$

Then

$$
\begin{aligned}
\left\|x_{n}^{\prime}\right\| & =\max _{0 \leq t \leq 1}\left|x_{n}^{\prime}(t)\right| \\
& =\max _{0 \leq t \leq 1}\left|\int_{0}^{t} x_{n}^{\prime \prime}(s) \mathrm{d} s\right| \\
& =\max _{0 \leq t \leq 1}\left|\int_{0}^{t}\left[f_{n}\left(s, x_{n}(s)\right)+e(s)-m^{2}(s) x_{n}(s)\right] \mathrm{d} s\right| \\
& \leq \int_{0}^{1}\left[f_{n}\left(s, x_{n}(s)\right)+e(s)\right] \mathrm{d} s+\int_{0}^{1} m^{2}(s) x_{n}(s) \mathrm{d} s \\
& =2 \int_{0}^{1} m^{2}(s) x_{n}(s) \mathrm{d} s \\
& \leq 2 \bar{m}^{2} r=: H .
\end{aligned}
$$

The fact $\left\|x_{n}\right\| \leq r$ and (3.19) show that $\left\{x_{n}\right\}_{n \in N_{0}}$ is a bounded and equicontinuous family on $[0,1]$. Now the Arzela-Ascoli Theorem guarantees that $\left\{x_{n}\right\}_{n \in N_{0}}$ has a subsequence, $\left\{x_{n_{k}}\right\}_{k \in \mathbb{N}}$, converging uniformly on $[0,1]$ to a function $x \in C[0,1]$. From the fact $\left\|x_{n}\right\| \leq r$ and (3.18), $x$ satisfies $\sigma r_{1} \leq x(t) \leq r$ for all $t \in[0,1]$. Moreover, $x_{n_{k}}$ satisfies the integral equation

$$
x_{n_{k}}(t)=\int_{0}^{1} G(t, s)\left[f_{n}\left(s, x_{n_{k}}(s)\right)+e(s)\right] \mathrm{d} s
$$

Let $k \rightarrow \infty$, and we arrive at

$$
x(t)=\int_{0}^{1} G(t, s)[f(s, x(s))+e(s)] \mathrm{d} s,
$$

where the uniform continuity of $f(t, x)$ on $[0,1] \times\left[\sigma r_{1}, r\right]$ is used. Therefore, $x$ is a positive solution of boundary value problem (1.4). Finally, it is not difficult to show that, $\|x\|<r$.

By Theorem 3.1, we have the following Corollary. 
Corollary 3.3. Let $\left(H_{0}\right)$ hold. Assume that there exist continuous functions $\bar{b}, b>0$ and $\lambda>0$ such that

$$
\text { (F) } 0 \leq \bar{b}(t) / x^{\lambda} \leq f(t, x) \leq b(t) / x^{\lambda} \text {, for all } x>0 \text { and } t \in[0,1] \text {. }
$$

Then problem (1.4) has at least one positive solution if one of the following two conditions holds:

(i) $e_{*} \geq 0$;

(ii) $e^{*}<0, \quad \bar{b}_{*}+\left(B^{*} / \sigma^{\lambda}\right)^{\lambda / \lambda+1} e_{*}>0$, where $B(t)=\int_{0}^{1} G(t, s) b(s) d s$.

Remark 3.4. When $m(t) \equiv m, t \in[0,1]$, then (1.4) reduces to (1.1), $\left(H_{0}\right)$ reduce to $m \in$ $(0, \pi / 2)$. So Corollary 3.3 is more extensive than [5, Corollary 3.1].

\section{Example}

Consider second-order Neumann boundary value problem

$$
\begin{gathered}
x^{\prime \prime}(t)+\left[\frac{\pi}{12}(3-t)\right]^{2} x(t)=\sqrt{2} t^{15}\left[x^{-1}(t)+1\right], \quad t \in(0,1), \\
x^{\prime}(0)=0, \quad x^{\prime}(1)=0 .
\end{gathered}
$$

Here , $f(t, x)=\sqrt{2} t^{15}\left[x^{-1}+1\right],(t, x) \in[0,1] \times(0,+\infty), e(t) \equiv 0, m(t)=(\pi / 12)(3-t), t \in[0,1]$. Obviously, $\left(H_{0}\right)$ is satisfied. Let $k(t)=\sqrt{2} t^{15}, g(x)=1 / x, h(x) \equiv 1, \phi_{r}(t)=\sqrt{2} t^{15} / 2, r=2$, then we can check that $\left(H_{1}\right),\left(H_{3}\right)$, and $\left(H_{4}\right)$ are satisfied. In addition, for $r=2$, we have

$$
\frac{r-r^{*}}{g(\sigma r)(1+h(r) / g(r))}=\frac{4 \sigma}{3}=\frac{4}{3} \frac{\min _{0 \leq t, s \leq 1} G(t, s)}{\max _{0 \leq t, s \leq 1} G(t, s)} \geq \frac{4 \cos ^{2} \bar{m}}{3}=\frac{2}{3}
$$

On the other hand, by Lemma 2.1, we have

$$
\psi^{\prime}(0)=\int_{0}^{1} m^{2}(t) \psi(t) \mathrm{d} t \geq \underline{m}^{2} \cos \bar{m}=\frac{\sqrt{2} \pi^{2}}{72}
$$

By (4.3), we have

$$
K^{*}=\max _{0 \leq t \leq 1} \int_{0}^{1} G(t, s)\left(\sqrt{2} s^{15}\right) \mathrm{d} s \leq \frac{\sqrt{2}}{16 \psi^{\prime}(0)} \leq \frac{9}{2 \pi^{2}}<\frac{2}{3} .
$$

Hence, $r-r^{*} /(g(\sigma r)(1+h(r) / g(r)))>K^{*}$. So that $\left(H_{2}\right)$ is satisfied. According to Theorem 3.1, the boundary value problem (4.1) has at least one positive solution $x$ with $0<\|x\|<2$.

For boundary value problem (4.1), however, we cannot obtain the above conclusion by Theorem 3.1 of paper [5] since $m(t)=(\pi / 12)(3-t), t \in[0,1]$ is not a constant. These imply that Theorem 3.1 in this paper complement and improve those obtained in [5]. 
International Journal of Differential Equations

\section{References}

[1] Y. Sun, Y. J. Cho, and D. O'Regan, "Positive solutions for singular second order Neumann boundary value problems via a cone fixed point theorem," Applied Mathematics and Computation, vol. 210, no. 1, pp. 80-86, 2009.

[2] A. Cabada and R. R. L. Pouso, "Existence result for the problem $\left(\phi\left(u^{\prime}\right)\right)^{\prime}=f\left(t, u, u^{\prime}\right)$ with periodic and Neumann boundary conditions," vol. 30, no. 3, pp. 1733-1742.

[3] A. Cabada, P. Habets, and S. Lois, "Monotone method for the Neumann problem with lower and upper solutions in the reverse order," Applied Mathematics and Computation, vol. 117, no. 1, pp. 1-14, 2001.

[4] A. Canada, J. A. Montero, and S. Villegas, "Liapunov-type inequalities and Neumann boundary value problems at resonance," Mathematical Inequalities and Applications, vol. 8, no. 3, pp. 459-475, 2005.

[5] J. F. Chu, Y. G. Sun, and H. Chen, "Positive solutions of Neumann problems with singularities," Journal of Mathematical Analysis and Applications, vol. 337, no. 2, pp. 1267-1272, 2008.

[6] D. Jiang and H. Liu, "Existence of positive solutions to second order Neumann boundary value problems," Journal of Mathematical Research and Exposition, vol. 20, no. 3, pp. 360-364, 2000.

[7] N. Yazidi, "Monotone method for singular Neumann problem," Nonlinear Analysis, vol. 49, no. 5, pp. 589-602, 2002.

[8] J.-P. Sun and W.-T. Li, "Multiple positive solutions to second-order Neumann boundary value problems," Applied Mathematics and Computation, vol. 146, no. 1, pp. 187-194, 2003.

[9] L. H. Erbe and H. Wang, "On the existence of positive solutions of ordinary differential equations," Proceedings of the American Mathematical Society, vol. 120, no. 3, pp. 743-748, 1994.

[10] P. B. Bailey, L. F. Shampine, and P. E. Waltman, Nonlinear Two Point Boundary Value Problems, Academic Press, New York, NY, USA, 1968

[11] D. J. Guo and V. Lakshmikantham, Nonlinear Problems in Abstract Cones, Academic Press, Boston, Mass, USA, 1988. 


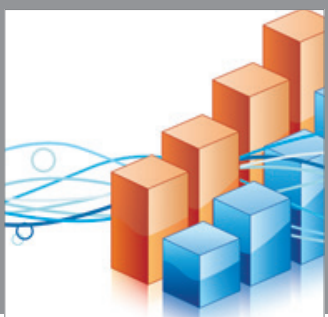

Advances in

Operations Research

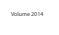

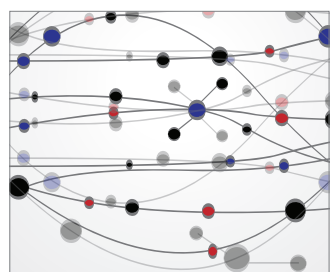

\section{The Scientific} World Journal
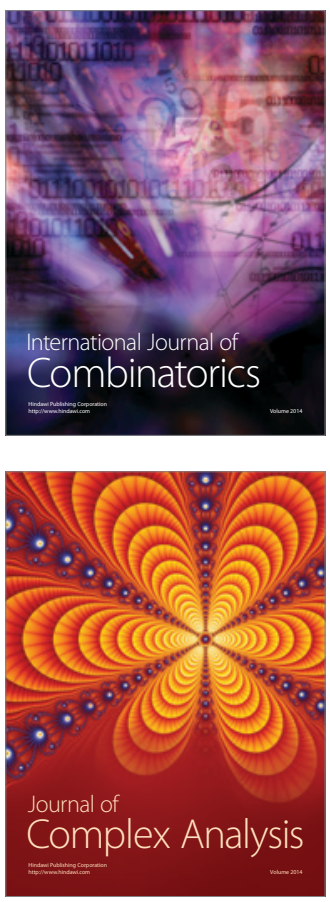

International Journal of

Mathematics and

Mathematical

Sciences
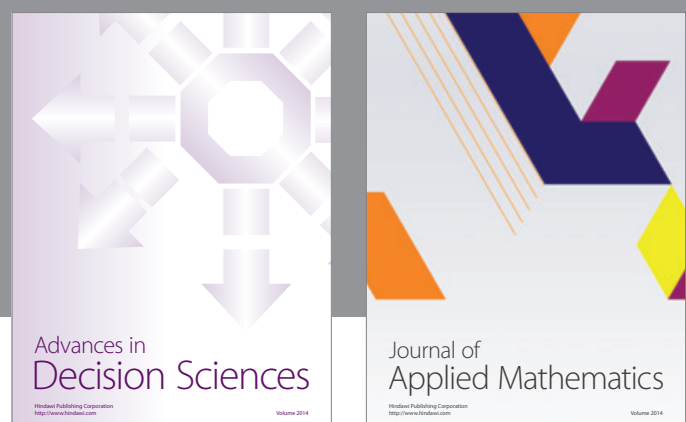

Journal of

Applied Mathematics
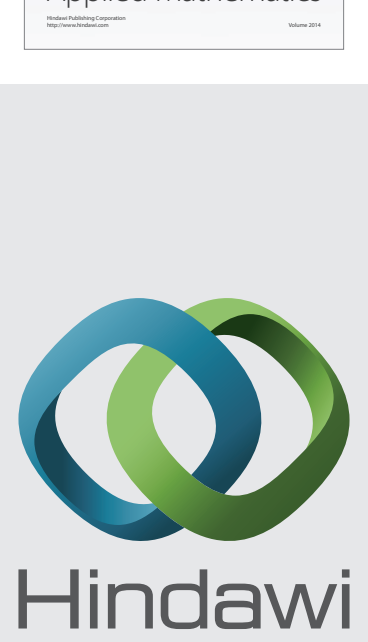

Submit your manuscripts at http://www.hindawi.com
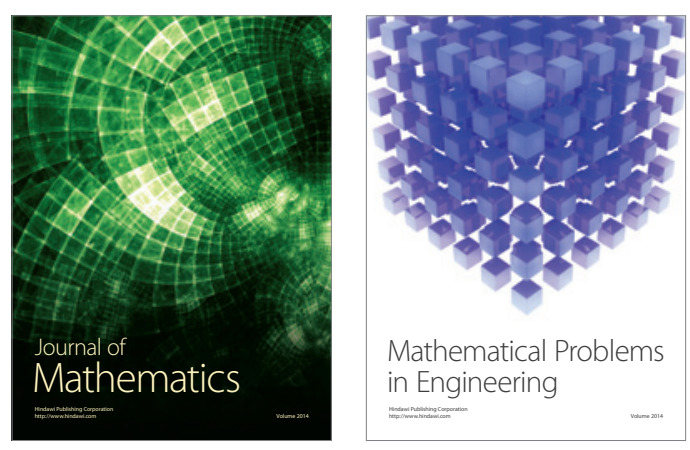

Mathematical Problems in Engineering
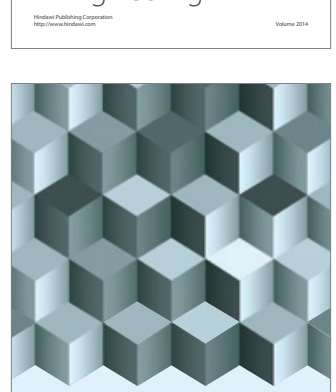

Journal of

Function Spaces
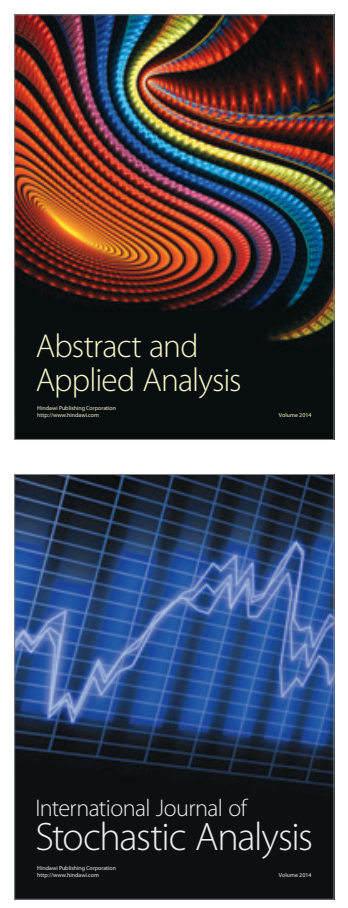

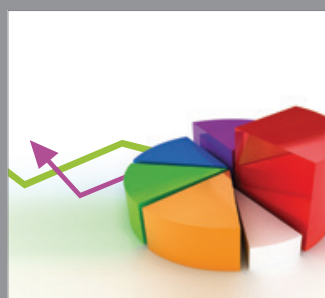

ournal of

Probability and Statistics

Promensencen
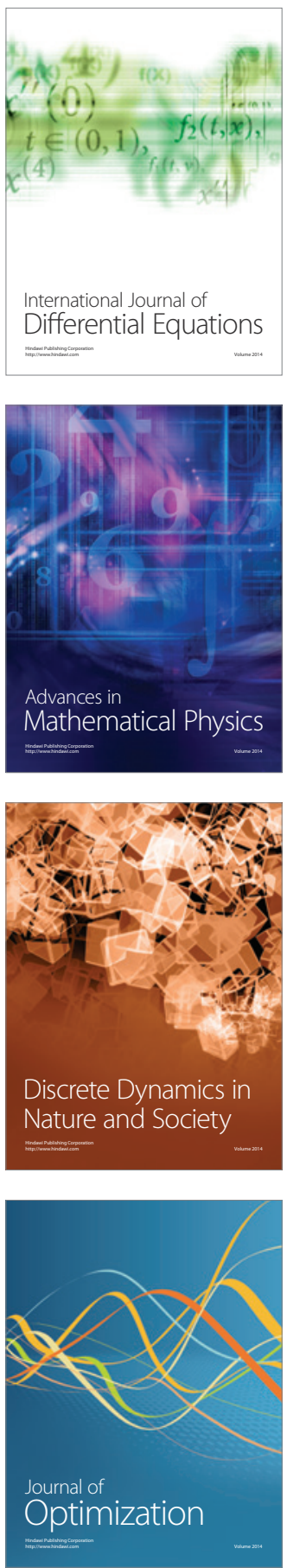